\title{
Assessment of consumer VR-headsets' objective and subjective field of view (FoV) and its feasibility for visual field testing
}

\author{
Yannick Sauer $^{1}\left(\mathbb{D} \cdot\right.$ Alexandra Sipatchin $^{1} \cdot$ Siegfried Wahl $^{1,2} \cdot$ Miguel García García $^{1}$
}

Received: 7 April 2021 / Accepted: 19 December 2021 / Published online: 15 January 2022

(C) The Author(s) 2022

\begin{abstract}
Virtual reality as a research environment has seen a boost in its popularity during the last decades. Not only the usage fields for this technology have broadened, but also a research niche has appeared as the hardware improved and became more affordable. Experiments in vision research are constructed upon the basis of accurately displaying stimuli with a specific position and size. For classical screen setups, viewing distance and pixel position on the screen define the perceived position for subjects in a relatively precise fashion. However, projection fidelity in HMDs strongly depends on eye and face physiological parameters. This study introduces an inexpensive method to measure the perceived field of view and its dependence upon the eye position and the interpupillary distance, using a super wide angle camera. Measurements of multiple consumer VR headsets show that manufacturers' claims regarding field of view of their HMDs are mostly unrealistic. Additionally, we performed a "Goldmann" perimetry test in VR to obtain subjective results as a validation of the objective camera measurements. Based on this novel data, the applicability of these devices to test humans' field of view was evaluated.
\end{abstract}

Keywords Virtual reality $\cdot$ Field of view $\cdot$ HMD $\cdot$ Perimetry $\cdot$ Eye relief $\cdot$ Visual field $\cdot$ Eye

\section{Introduction}

Virtual reality research has been conducted for over 25 years (Cipresso et al. 2018). During this period, the technical specifications from original devices have fallen far behind the present ones. Greater resolutions, bigger fields of view (FoV), the introduction of eye-tracking technologies and significantly higher computational power have improved the overall virtual reality experience. All of these vast enhancements have led to novel applications in a variety of fields such as medical training (Bric et al. 2016; Pfandler et al. 2017), military training (Alexander et al. 2017) or gaming (Zyda 2005). Likewise, further experimental possibilities in psychophysics and vision sciences have been opened (Tychsen and Foeller 2020; Mohamed Elias et al. 2019; Tieri et al. 2018) as these devices became more and more affordable. Moreover, there has been a notable rise

Yannick Sauer

yannick.sauer@uni-tuebingen.de

1 Institute for Ophthalmic Research, University of Tuebingen, Elfriede-Aulhorn-Straße 7, 72072 Tübingen, Germany

2 Carl Zeiss Vision International GmbH, Turnstraße 27, 73430 Aalen, Germany in clinical applications of VR, led by telemedicine systems for home-based medical assessments (Bashshur et al. 2020; Tatiyosyan et al. 2020; Neugebauer et al. 2021) where, for example, different ophthalmological (visual-field) tests have been proposed (Hollander et al. 2000; Tsapakis et al. 2017, 2018; Mees et al. 2020).

In virtual reality, immersion refers to the process of losing awareness of the simulated world's virtuality and accepting it as real. More immersive environments have reported zeal and alacrity toward the performed task and better assimilation of concepts. Hence, every developer attempts to improve the immersion by tweaking the virtual environment. However, some factors that affect the immersion feeling are related to the hardware used rather than the developed software. Among these, the FoV that head-mounted displays (HMDs) may reach can greatly impact the perception of the virtual environment (Miller and Bugnariu 2016; Hollander et al. 2000) and can limit possible applications for virtual reality.

Thus, it is not strange that head-mounted display manufacturers have entered a fierce competition to have the greatest field of view displaying the fewest aberrations. Manufacturers' claims may refer to the rendered FoV, without considering how eye relief (frontal distance of eyes to 
HMD), inter-pupillary distance (IPD) or lens to display distance, factors that actually compromise the effective field of view.

The FoV size promised by manufacturers, if not fully visible, might not only compromise the user's experience but it can also introduce an instrumental error in experiments that rely on a precise size and positioning of their stimulus. For instance, measuring visual field defects requires precise knowledge of which FoV actually can be assessed. Moreover, when studying visual perception in virtual reality, knowledge about the size and shape of the FoV can be important. For example, distance perception (Masnadi et al. 2021), navigation (Hassan et al. 2007) and speed and motion perception (Caramenti et al. 2019; Segawa et al. 2012; Warren and Kurtz 1992; Pretto et al. 2009) have been shown to depend on the FoV size. One recent study showed a strong FoV dependence of perceived heading from distorted optic flow in VR (Sauer et al. 2021), so that individual FoV differences between subjects might have influenced the experimental results. To know which part of the visual field was exposed to optic flow and to guarantee comparability between different subjects in VR-based motion perception studies, an objective measurement of the effective FoV is required.

Here we introduce an easy and affordable method to quantify the size and shape of the FoV and estimate the influence of individual eye position parameters. This setup allows fast validation of the official specifications and opens the possibility to compare different headsets. The used camera equipment (Raspberry Pi and fisheye camera) is inexpensive and available for the consumer market.

The method presented here was tested by measuring the FoV of multiple generic HMDs. To compare the camerabased FoV measurements to the one perceived by subjects, we recreated an established clinical field of view test (perimetry) in virtual reality. In this test, the subject has to respond about the visibility of a moving peripheral stimulus. The answers help to delimit the individual's FoV along multiple meridians of the visual field. We further conducted a classical semi-automated FoV test (Octopus 900, Haag Streit International, Koeniz, Switzerland) to compare the virtual reality recreated test to the standardized clinical procedure.

\subsection{Related work}

Often, the FoV of different HMDs is compared directly using their rendered FoV, as in the database by Musil (2021). Rendered values can be easily extracted from the VR subsystem as implemented by Musil. However, as recognized before by Pohl et al. (2016) not all parts of the HMD screen are visible. Furthermore, lens distortions can change the perceived position if not corrected accordingly (Rolland and Hopkins 1993; Martschinke et al. 2019) or the settings for
FoV of the virtual camera might not fit the physical FoV of the HMD. Therefore, rendered FoV cannot be taken as a measurement of visible FoV, and other objective measurements are necessary.

Wheelwright et al. (2018) suggested already camerabased FoV measurements. They also explained that individual face shape could influence FoV, yet they did not measure individual HMDs to compare with the manufacturers' specifications. Lynn et al. (2020) measured, among other physical properties, the lens diameter and screen size of different consumer headsets to calculate the horizontal FoV as the opening angle of the lens. This method does not consider the optical properties of the lenses and requires disassembling the headsets to measure screen distance. In contrast, our camera-based measurement can be used easily without disassembling and additionally shows the exact shape and area of the FoV. Furthermore, previous work did not measure the influence of different eye relief and IPD values on the exact size and shape.

\section{Apparatus}

Head mounted displays In this study, several virtual reality head-mounted displays were evaluated, from some developer kits, first customer commercially available ones, passing by the first HMD to embed eye-tracking, to a canted device which exhibits a great increase in the field of view. A list of the tested devices can be found below:

- StarVR One (StarVR, Taipei, Taiwan)

- HTC Vive Pro Eye (HTC Cooperation, Xindian, Taiwan)

- HTC Vive (HTC Cooperation, Xindian, Taiwan)

- Pico Neo 2 (Pico Interactive, San Francisco, CA, USA)

- Oculus Rift DK2 (Oculus VR, Irvine, CA, USA)

- Fove 0 (Fove Inc, Torrance, CA, USA)

Camera \& Set-up A camera-based measurement of the field of view of HMDs gives the possibility to precisely determine the size and shape of the visible FoV and examine the influence of different eye positions, horizontal and distance to the HMD lenses. We used a wide field of view fisheye Raspberry Pi camera (Joy-IT 5MP Wide-Angle Camera for Raspberry Pi (rb-camera-WW2), SIMAC Electronics GmbH, Neukirchen-Vluyn, Germany), which is small enough to be placed as close in the headset as eyes would be and with $200^{\circ} \mathrm{FoV}$ can capture the complete image presented in the headset, even for wide FoV HMDs.

To change vertical and horizontal alignment as well as the distance of the camera to the lens of the HMD, the relative position of the camera and HMD should be adjustable in all three directions. As illustrated in Fig. 1, the different HMDs were fixated with clamps on a stand mounted to an optical 


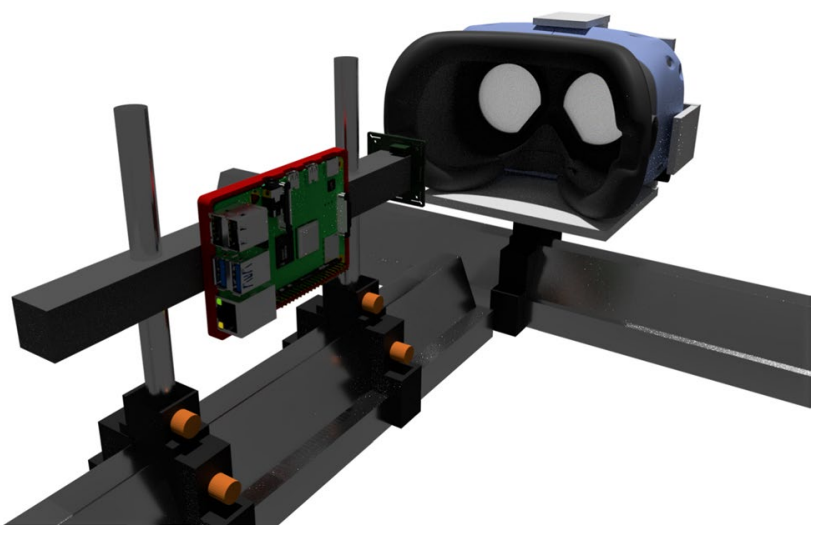

Fig. 1 Set-up for the camera-based measurement of visible FoV: HMDs were fixated with clamps on a stand that was movable on an optical rail. The camera connected to a Raspberry Pi was placed at the end of a rod which was mounted on an optical rail. The camera mount was height adjustable to align the camera for each HMD. Camera-lens distance was changed by moving the camera mount on the optical rail. Horizontal camera to lens alignment, representing different IPDs, was changed by moving the HMD on the other rail. Raspberry Pi model modified from Juanma de las Heras (grabcad. com). Headset model modified from HTC Vive Pro model by Eternal Realm (licensed under Creative Commons Attribution)

rail. The camera was placed on a second rail orthogonal to it. Both rails could then be used to change relative horizontal position as well as simulate different eye relief distances. For vertical alignment, the camera mount was height adjustable.

Hardware \& Software The virtual testing environments were constructed and tested using Unity 2019.4.18f1 (Unity Technologies, CA, USA) in a Windows 10 (20H2) PC with 16 GB of RAM, a processor i7-10700 and a graphics card Nvidia GeForce 3080. The scripts for capturing the images with the Raspberry Pi were written in Python, and all analysis was performed in Matlab (The Mathworks Inc, Natick, MA, USA).

\section{Methods}

\subsection{Camera calibration}

A precise camera calibration is needed to compensate for the camera's distortions and to recalculate the pixel positions in the captured images into angular position in the field of view. For calibration, pictures of a $7 \times 9$ checkerboard pattern with square size $20 \mathrm{~mm}$ were taken from various angles. Afterward, these images were used in the Urban et al. (2015) omnidirectional camera calibration toolbox, which is an improved version of Scaramuzza et al. (2006) OCamCalib Toolbox for MATLAB. In 74 images, the checkerboard pattern was successfully detected, and calibration was calculated with a mean reprojection error of $1.0025 \mathrm{px}$. The calibration can then be used to transform an image pixel position $(x, y)$ into a normalized vector $\left(x_{w}, y_{w}, z_{w}\right)$ pointing to the corresponding world location.

In this experiment, the camera's position is always measured relative to the front of the camera's lens. To determine the offset between the lens front and the camera's entrance pupil (the origin of the world vector $\left.\left(x_{w}, y_{w}, z_{w}\right)\right)$, a set of pictures of a pattern of known size were taken at multiple distances $d$ from 5 to $40 \mathrm{~mm}$. In each image, the pixel position of 25 points were transformed to their world vectors using the camera calibration. The known distance of the points in real world was then used to calculate the distance of the pattern to the entrance pupil $e$. The offset $\Delta d$ is the difference $e-d$. The mean overall measured distances was $\Delta d=(3.2 \pm 0.4) \mathrm{mm}$.

\subsection{Camera-based field of view measurement}

The above-mentioned camera setup was used to take images of the HMD's left eye view at different eye relief distances. The camera feed was shown on a separate screen and overlayed with a pattern of concentric circles around a cross at the pixel position of the optical axis, as returned by the camera calibration. At the same time, an alignment pattern (concentric circles of different colors) on top of a white background was displayed in the HMD. With the camera placed directly in front of the HMD, the central cross and the virtual image's center were aligned. Then, the orientation of the HMD was adjusted until the camera overlay pattern and HMD pattern stayed aligned when moving the camera along the orthogonal rail, simulating a straight gaze position at correctly aligned IPD. Pictures were taken every $5 \mathrm{~mm}$ from the HMD lens, up to $40 \mathrm{~mm}$. Different IPDs were also simulated by moving the HMD only horizontally out of the aligned position, without changing the orientation.

The process of calculating the FoV contour in visual angle from the captured images was performed entirely in MATLAB R2020b (The Mathworks, Inc., Natick, USA) using the Image Processing Toolbox as illustrated in Fig. 2. The images taken for FoV measurement were binarized. First, holes were closed in the binary image and then small areas removed, which mainly were caused by bright reflections on the lenses of the HMDs. Finally, edges were cleaned using morphological closing.

The contour of the remaining area in the binarized image is taken as the outline of the visible FoV. Using the camera calibration, the pixel coordinates $(x, y)$ of the contour were transformed to vectors $\left(x_{w}, y_{w}, z_{w}\right)$ pointing into the direction of the corresponding world position. Polar angle $\varphi$ and azimuthal angle $\theta$ are then calculated following Eq. 1. 
(A) Calibration image

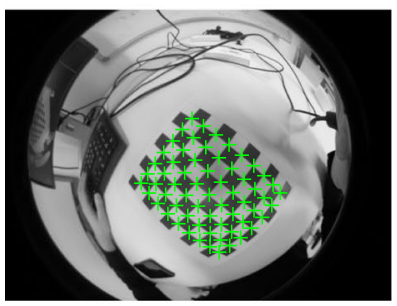

(B) Original image

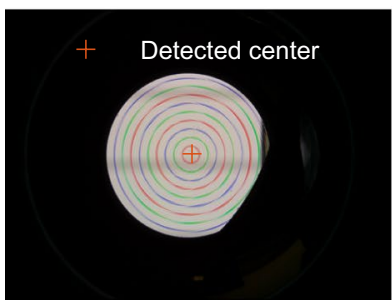

Calibration view points

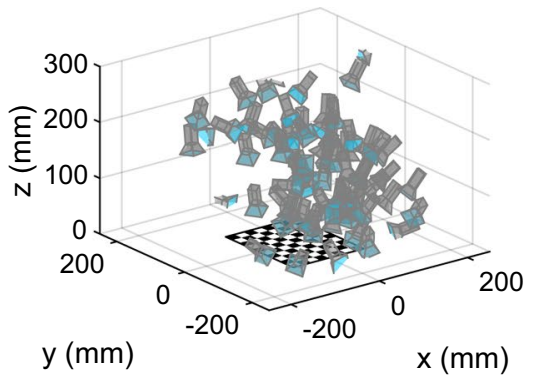

Binarized image

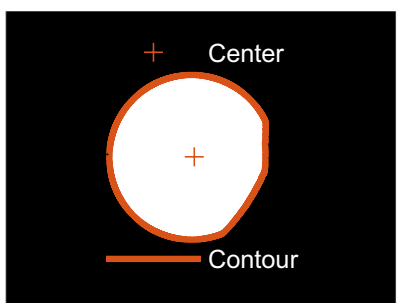

Undistorted calibration image
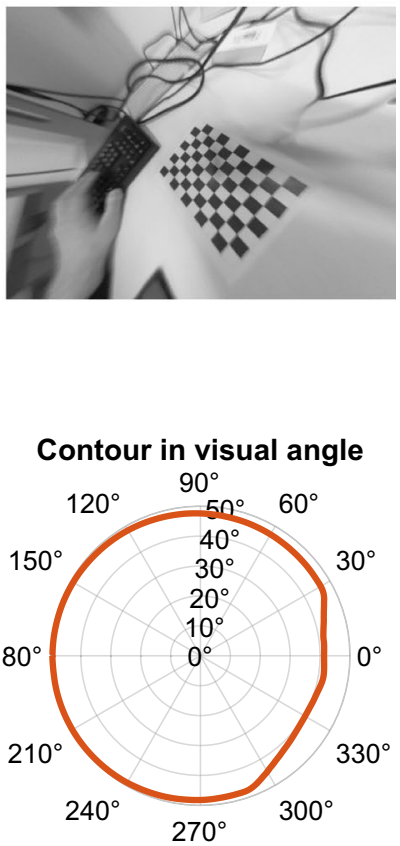

Fig. 2 A) Calibration procedure. From left to right, an example calibration image is shown with the marked detected checkerboard corners, a $3 \mathrm{~d}$ representation of the spatial camera locations for all calibration images and the resulting image after distortion correction. B)

$\left(\begin{array}{c}\varphi \\ \theta\end{array}\right)=\left(\begin{array}{r}\arctan \frac{x_{w}}{y_{w}} \\ \arctan \frac{\sqrt{x_{w}^{2}+y_{w}^{2}}}{z_{w}}\end{array}\right)$

The polar angle $\varphi$ is the angle of a meridian through the center of the visual field and $\theta$ the limitation of FoV in this direction. To compare HMDs, we extract vertical, nasal and temporal FoV size as the $\theta$ value for the vertical meridian and the horizontal half-meridians, respectively. The final field of view values are reported for each headset at the average corresponding eye relief distance.

Since the FoV shape might be different between HMDs, another measurement for comparison is the solid angle $\Omega$ of the area enclosed by the FoV, as defined by Eq. 2 .

$$
\begin{aligned}
\Omega & =\iint_{A} \mathrm{~d} \Omega=\int_{-\pi}^{\pi} \int_{0}^{\theta(\varphi)} \sin \theta \mathrm{d} \theta \mathrm{d} \varphi \\
& =\int_{-\pi}^{\pi}(1-\cos (\theta(\varphi))) \mathrm{d} \varphi
\end{aligned}
$$

This term was calculated by the summation of $(1-\cos (\theta(\varphi))) \mathrm{d} \varphi$ over all meridians.
Contour detection work flow: From left to right, original image with the detected center, binarized image with the contour defined, and final contour in visual angles

\subsection{Subjects}

Twenty-one (21) participants (13 males / 8 females) aged between 22 and 40 years (mean $=29$ years) took part in the study, and their interpupillary distance and eye relief for each headset were measured. None of the study participants reported any ocular complication or any medical condition that affected their normal vision or their motion judgements, such as cataracts, retinal or corneal dystrophies, epilepsy, or prior history of motion sickness.

\subsection{Eye relief distance}

The distance between the wearer's eyes and the HMD lenses (eye relief) varies due to the device's dimensions, and the way it fits to the physiognomy of the wearer's head. Moreover, it can also vary depending on how tightly the straps that hold the device are fastened and how soft the foam surrounding the face is.

Custom 3D printed models in the shape of a hollow cylinder (See Fig. 3 and Supplementary material A.1) were used along with small paper tubes to measure each subject's distance and get a general idea of what this distance might be for every device. Subjects wore the headset to which the hollow cylinder pieces were previously taped and, with their eyelids closed, pushed the paper tubes inside the cylinder 


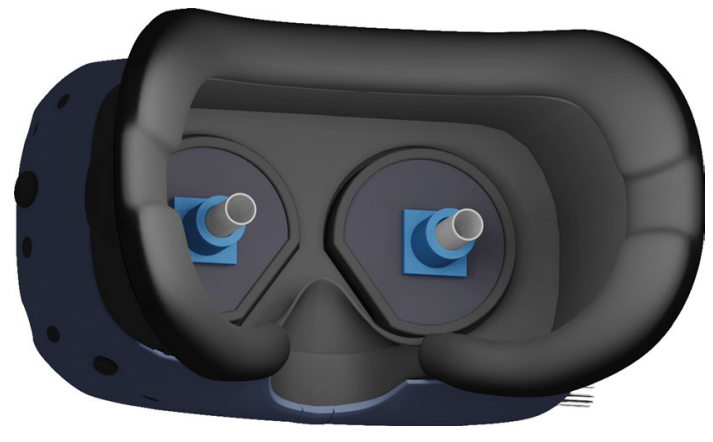

Fig. 3 Figure showing one type of the 3D printed models and how they are located. Modified from HTC Vive Pro model by Eternal Realm (licensed under Creative Commons Attribution)

Table 1 Results of eye relief measurements for all HMDs

\begin{tabular}{llll}
\hline HMD & $\begin{array}{l}\text { Mean eye relief } \\
(\text { e.r. })(\mathrm{mm})\end{array}$ & $\begin{array}{l}\text { SD } \\
(\mathrm{mm})\end{array}$ & $\begin{array}{l}\text { Range } \\
(\min .-\mathrm{max} .)(\mathrm{mm})\end{array}$ \\
\hline StarVR One & 18.2 & 1.7 & $15.8-23.9$ \\
HTC Vive Pro & 17.0 & 2.2 & $14.5-24.6$ \\
HTC Vive & 16.6 & 1.9 & $14.4-23.0$ \\
Pico Neo & 18.3 & 2.1 & $14.8-23.2$ \\
Oculus Rift DK2 & 10.7 & 0.9 & $8.6-13.6$ \\
Fove 0 & 11.4 & 1.7 & $8.4-15.9$ \\
\hline
\end{tabular}

holes as they fastened the headsets. When finished, the device was removed, and the distance was measured using a caliper. These measurements were repeated twice per eye and headset.

This separation can be referred to as the closed eye relief distance, yet this distance can vary from the natural eye relief due to the upper eyelid thickness (Osuobeni and AlMijalli 1997; Lee et al. 2006). However, at the same time, the eye translates backward when it is closed (Kirchner et al. 2021), and it can be even retracted further due to the pressure applied by the piece of paper. Given the uncertainty on this parameter, eye relief was not modified from the measured value. Likewise, the distance to the eye's entrance pupil was neglected for the FoV calculations as the value roughly matches the fisheye camera aperture entrance.

For every HMD, the typical range of eye relief was determined by taking the mean of all measurements of all 21 subjects combined. Results are presented in Table 1 . The fields of view for specific eye relief values were linearly interpolated from the measurements taken between 5 and $40 \mathrm{~mm}$.

\subsection{Interpupillary distance (IPD)}

For most of the HMDs used in this study, the position of the lenses was fixed in the headset. Only for the HTC Vive and HTC Vive Pro do changes to the IPD settings also change horizontal distance between the lenses. For the other HMDs, changes to IPD settings adjust only the rendered image, by adapting both eye's projection matrices.

IPD of every subject was measured manually using a pupilometer (Breitfeld \& Schlikert GmbH, Karben, Germany) and the eye tracking-based automatic IPD detection algorithms from the headsets. The mean IPD found in our subjects was $63.9 \mathrm{~mm}$ and ranged from 58 to $73 \mathrm{~mm}$.

When possible, the lenses of the devices were adjusted manually to fit the subject's IPD. This way, subjects always obtained the exact center of the image when the IPD was set correctly, and the limits of the FoV only shifted horizontally. The effect of different IPDs was analyzed for the StarVR One, the HTC Vive Pro and the Fove 0. For the first ones, pictures of a small, default and big IPD were taken at the average eye relief distance. As the HTC Vive Pro can be manually adjusted to fit individual IPD, photographs were taken either with the lens distance adjusted for correct IPD alignment or while the headset remained at the default IPD settings. For comparison, pictures with a headset without IPD correction possibilities (Fove 0 ) were taken.

\subsection{Binocular FoV}

It is assumed that the right eye's field of view is the mirrored version of the left one. The combined binocular FoV along the horizontal meridian is then calculated by doubling the larger angle from either the temporal or nasal direction. To get the binocular contour, the monocular field of views are merged by taking the maximum value from either eye along each meridian. Binocular overlap is the region of the binocular FoV which is seen by both eyes at the same time. As quantification, we calculate the ratio between the overlapped area and the binocular area. These calculations are based on the assumption of perfect IPD alignment, in which case the subject's visual field center for straight gaze is aligned with the headset's FoV center.

\section{Results}

Figure 4 shows the FoVs obtained for each headset at their normal eye relief distances, for a direct comparison. In Fig. 5, individual panels for each HMD show the influence of different eye relief values. Table 2 displays the FoV values extracted from these contours.

Effect of eye relief As mentioned in the Sect. 3.4, eye relief varies among headsets. This distance is in fact significantly different $(p=0.009, \mathrm{~K}-\mathrm{W})$ with pair-wise differences between the Oculus Rift DK2 (smallest eye relief distance) and the rest of headsets, except for the Fove 0 and the original HTC Vive. Fove 0 also differs from the Pico Neo 2 (biggest eye relief). 


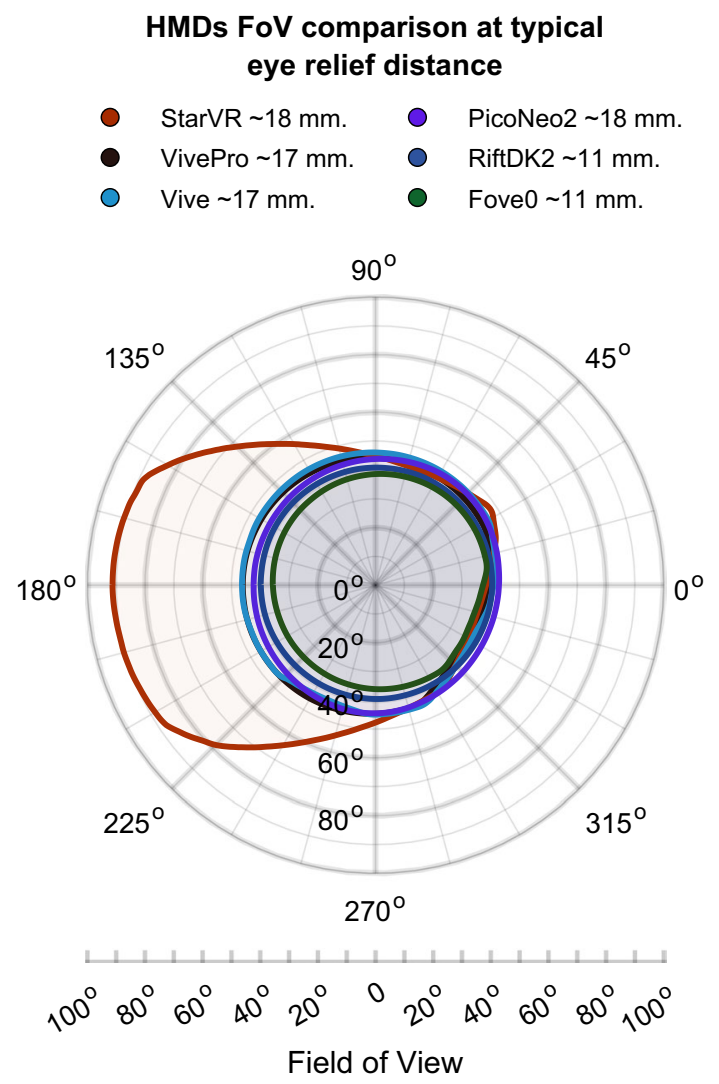

Fig. 4 This figure shows the different field of views achieved by each headset at approximately their eye relief distances

In most cases, the size and shape of the lens determine the FoV, meaning the outer parts of the screen are not visible through the lens. An increase in eye relief will, therefore, always reduce the FoV size. For small eye relief, also the screen size becomes relevant. For Vive and Vive Pro, for example, the nasal FoV is limited by the screen for the minimum eye relief, a vertical cut-off is visible. At mean eye relief distance, only for Oculus Rift DK2 and Fove 0 the screen size limits the FoV.

Effect of IPD FoV measurements for fixed eye relief at different IPDs are shown in Fig. 6.

For the StarVR One, the nasal FoV changes minimally with IPD $\left(<1^{\circ}\right)$. Only temporal FoV shifted 4 degree between minimum IPD $53 \mathrm{~mm}$ and maximum IPD $77 \mathrm{~mm}$.

When manually adjusting the lens position of the HTC Vive Pro, the field of view stays roughly the same (change of nasal and temporal $<2^{\circ}$ between IPDs $61 \mathrm{~mm}$ and $70 \mathrm{~mm}$ ).

Simulating a misaligned headset, meaning the lens distance was set to a fixed value $64 \mathrm{~mm}$, there is a difference of $11^{\circ}$ in the temporal FoV between smallest and biggest IPD. The nasal FoV shows only a small difference of $2.4^{\circ}$, as the field of view here reaches the limit of the screen.

There are also big differences for the Fove 0 , where lens position cannot be adjusted. Temporal FoV changes by $11.8^{\circ}$, nasal by $5.6^{\circ}$ between the different IPD values $60 \mathrm{~mm}$ and $69 \mathrm{~mm}$. The limit of the nasal shift is due to the screen size.

\section{VR perimetry}

In 1945, Hans Goldmann introduced a hemispherical bowl perimeter, which soon became the "gold standard" in clinical practice (Goldmann 1946). This device allowed a precise measure of the visual field boundaries of each participant. Although the perimeters evolved and fully or semi-automated versions measuring the whole field of view appeared, Goldmann's perimeter idea remains active in clinical use. This part of the experiment aims to observe the feasibility of performing virtual perimetry based on the Goldmann's device, albeit with the benefits and limitations of virtual reality headsets.

Two options may appear, as the subject's field of view might be delimited by either the HMD's FoV limits or the eye's visual field limits.

Limits of the FoV were obtained by moving a stimulus along different meridians separated by $15^{\circ}$, while participants fixated at a central fixation target; this procedure is usually referred to as kinetic perimetry. The moving stimulus was created in Unity, as a sphere located at the surface of a virtual sphere with a radius of $50 \mathrm{~m}$ and whose center was at the eye (virtual camera). The stimulus subtended $0.8^{\circ}$ of visual angle at all times. For monocular presentation, the virtual camera was set to render to the left eye only. The virtual FoV of the camera was set automatically by the VR plugin in Unity. First, the stimulus moved outward. Subjects were instructed to press a button as soon as the moving target was not visible to them anymore. Then, the stimulus was set further outside and moved inward until subjects pressed again, indicating its reappearance in their visual field. This procedure of moving outward and inward was repeated three times for each meridian. The subjective FoV along each meridian was calculated as the mean of those six measurements.

Unlike traditional testing, some of these HMDs embed eye-tracking technologies, which allow for a gaze contingency paradigm (Sipatchin et al. 2021; Stein et al. 2021), ensuring that the subject always fixates at the center by interrupting the test when gaze was not on the fixation target.

\subsection{Luminance limits}

Objects moving in the periphery while gaze is fixed centrally might need a larger size or brightness to be detected by our visual system. Likewise, for some psychophysical experiments, the stimulus's luminance and contrast have to be carefully controlled. However, this is not usually the case in virtual reality, where each headset has different display panels (even different display panel technologies). 


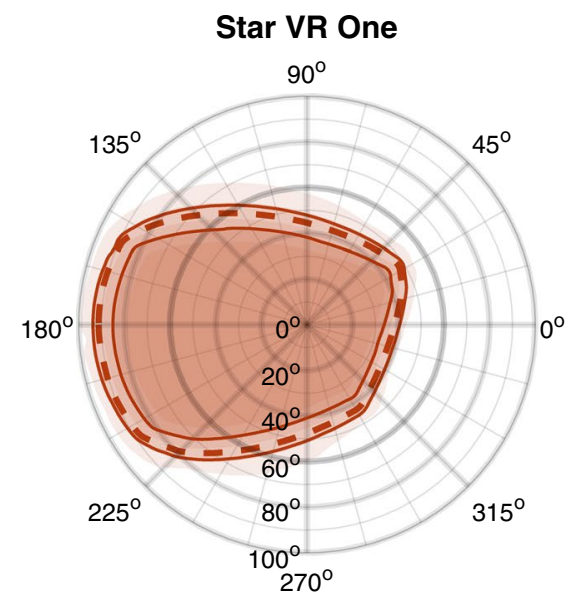

Pico Neo 2

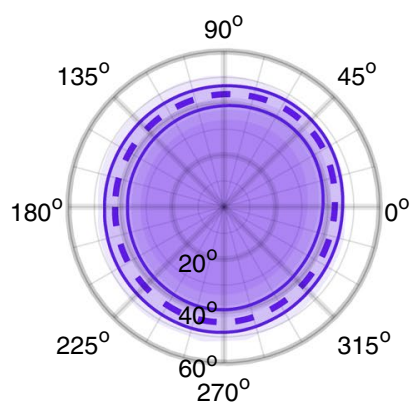

HTC Vive Pro

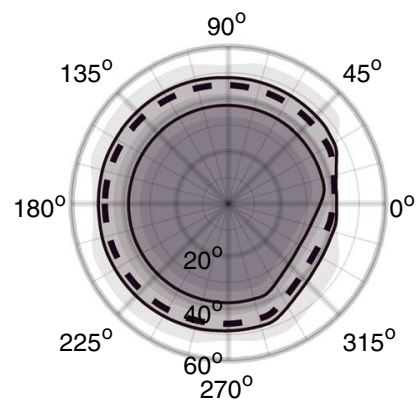

Oculus Rift DK2

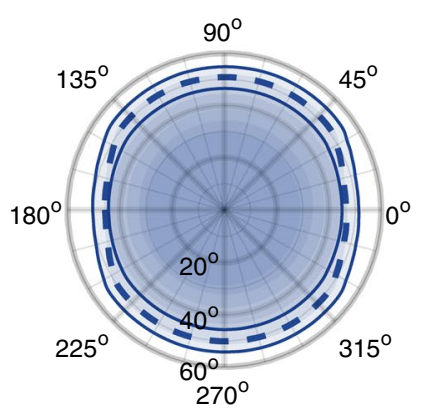

HTC Vive

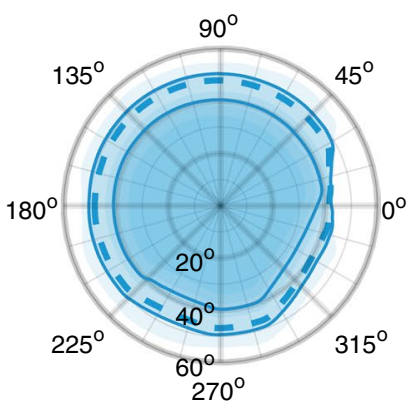

Fove 0

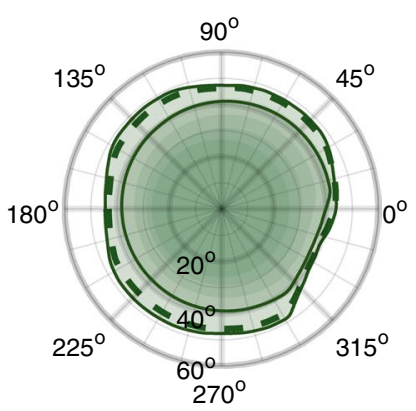

Fig. 5 Overview of field of view range for all measured HMDs: The dashed contour is the FoV for the measured mean eye relief. Solid contours represent the FoV for the minimum and maximum eye relief of our subjects for each HMD. All figures show the left eye's FoV.
The gradient area indicates the FoV measured at different camera to lens distances from the brighter (closest) to the more opaque ones (farthest) in a range from 10 to $30 \mathrm{~mm}$
Table 2 Overview of FoV extents at mean eye relief for each HMD

\begin{tabular}{|c|c|c|c|c|c|c|c|c|}
\hline \multirow[t]{2}{*}{ HMD } & \multirow[t]{2}{*}{ Vertical } & \multicolumn{2}{|c|}{ Monocular $\left({ }^{\circ}\right)$} & \multicolumn{2}{|c|}{ Binocular $\left(^{\circ}\right)$} & \multicolumn{2}{|c|}{$\begin{array}{l}\text { Solid angle } \\
(\Omega)\end{array}$} & \multirow{2}{*}{$\begin{array}{l}\text { Binocular } \\
\text { overlap } \\
(\%)\end{array}$} \\
\hline & & Temporal & Nasal & Horizontal & Maximum & ${ }^{(2)}$ & $\left(\mathrm{rad}^{2}\right)$ & \\
\hline StarVR One & 92 & 91 & 38 & 182 & $182 @ 0^{\circ}$ & 9854 & 3.00 & 37 \\
\hline HTC Vive Pro & 91 & 47 & 40 & 94 & $94 @ 0^{\circ}$ & 5937 & 1.81 & 84 \\
\hline HTC Vive & 95 & 48 & 42 & 96 & $96 @ 0^{\circ}$ & 6322 & 1.93 & 88 \\
\hline Pico Neo 2 & 88 & 42 & 43 & 86 & $88 @ 71^{\circ}$ & 5572 & 1.70 & 96 \\
\hline Oculus Rift DK2 & 101 & 46 & 47 & 94 & $102 @ 42^{\circ}$ & 7192 & 2.19 & 98 \\
\hline Fove 0 & 92 & 43 & 43 & 86 & $93 @ 60^{\circ}$ & 6034 & 1.84 & 95 \\
\hline
\end{tabular}

Vertical, temporal and nasal FoV were extracted from the monocular FoV contours. For the combined binocular FoV, we give values for the horizontal meridian as well as the maximum FoV, which is along a diagonal meridian for some HMDs. Solid angle $\Omega$ was determined for the binocular FoV contour. From this, the overlap between the eyes was calculated, as the ratio of overlapping FoV to the combined binocular FoV
In order to define the perimeter of the eye's field of view, the background and stimulus luminance have to be controlled. For this reason, luminance values for each headset were measured.

The virtual environment's skybox was fixed at Hue and Saturation, while Value was increased from 0 to 100 (in steps of 10). At each step, three luminance measurements were taken using the luminance meter (Konica Minolta LS-110, Konica Minolta, Inc., Tokyo, Japan) with the close-up lens in a dark room.

The results from the luminance measurements can be found in the Table 3 and Fig. 7.

Only the HTC Vive and Pro version could present an equivalent to the stimulus $3\left(100 \mathrm{~cd} \mathrm{~m}^{-2}\right)$. Therefore, and in 


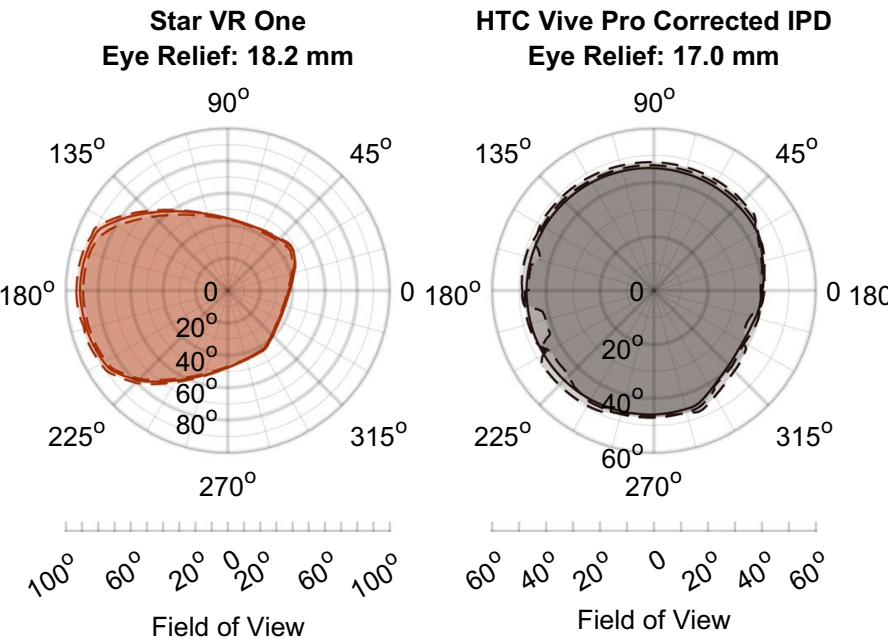

Field of View
HTC Vive Pro Misaligned

Eye Relief: $17.0 \mathrm{~mm}$

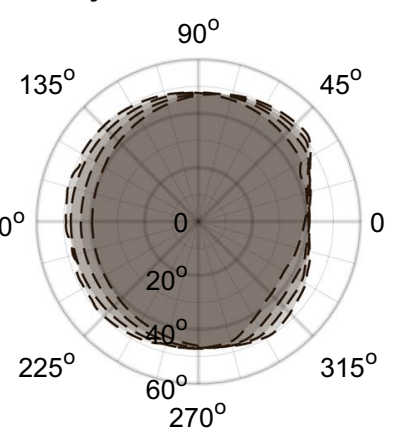

$60^{\circ} \quad 40^{\circ} \quad 20^{\circ} \quad 2 \quad 20^{\circ} \quad 40^{\circ} \quad 60^{\circ}$ Field of View
Fove 0

Eye Relief: $11.4 \mathrm{~mm}$

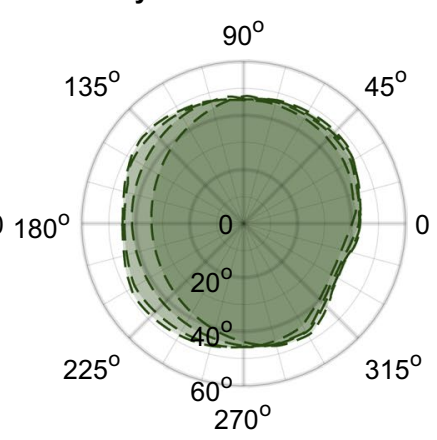

$60^{\circ} \quad 40^{\circ} \quad 20^{\circ} \quad \circ \quad 20^{\circ} \quad 40^{\circ} \quad 60^{\circ}$

Field of View

Fig. 6 Figure showcasing the influence of different inter-pupillary distances on the FoV. From left to right, StarVR One (no physical adjustment + canted display), HTC Vive Pro with the IPD adjusted

Table 3 Results of "Value" in HSV Color space for the different stimulus luminance in the Goldmann

\begin{tabular}{|c|c|c|c|c|c|}
\hline \multirow[t]{2}{*}{ HMD/values } & \multirow[t]{2}{*}{ Background } & \multicolumn{2}{|c|}{ Stimulus } & \multirow{2}{*}{$\begin{array}{l}\operatorname{Max} \\
\left(\mathrm{cd} \mathrm{m}^{-2}\right)\end{array}$} & \multirow[t]{2}{*}{ Min } \\
\hline & & (2) & (3) & & \\
\hline Goldmann & $10 \mathrm{~cd} \mathrm{~m}^{-2}$ & 32 & 100 & - & - \\
\hline (Luminance) & $31.5 \mathrm{asb}$ & $\mathrm{cd} \mathrm{m}^{-2}$ & $\mathrm{~cd} \mathrm{~m}^{-2}$ & & \\
\hline HTC Vive & 28.8 & 46.5 & 76.81 & 178.7 & 0.0 \\
\hline HTC Vive Pro & 33.8 & 55.6 & 93.4 & 116.1 & 0.0 \\
\hline StarVR One & 42.6 & 70.8 & o.o.r. & 68.4 & 0.0 \\
\hline Oculus Rift DK2 & 39.9 & 71.9 & o.o.r. & 62.4 & 0.0 \\
\hline Pico Neo 2 & 43.5 & 74.0 & o.o.r. & 60.5 & 0.1 \\
\hline Fove 0 & 29.1 & 55.3 & o.o.r. & 97.2 & 0.1 \\
\hline
\end{tabular}

“o.o.r." stands for out of range

order to be comparable with traditional testing, our stimulus was set to $I V 2 e$ in the Goldmann scale, defined as a stimulus size of $0.8^{\circ}$ visual angle and luminance of $32 \mathrm{~cd} \mathrm{~m}^{-2}$. The background was defined a grey hemisphere of size $50 \mathrm{~m}$ and luminance $10 \mathrm{~cd} \mathrm{~m}^{-2}$ or $31.5 \mathrm{asb}$.

\subsection{Comparison with Octopus perimeter}

Virtual perimetry was measured with two different headsets (HTC Vive Pro and the StarVR One), with Tobii eyetracking technologies. A subset of six subjects from our original subject pool (mean IPD $=66.6 \mathrm{~mm}$; ranging from 61 to $73 \mathrm{~mm}$ ) undertook the virtual perimetry test in both headsets with their individual eye relief and correct IPD settings. The mean eye relief values of these six subjects were $18.04 \mathrm{~mm}(16.6-23.1 \mathrm{~mm})$ and $18.33 \mathrm{~mm}(16.3-22.3 \mathrm{~mm})$ for the StarVR and for the HTC Vive Pro, respectively. using the knob to match the IPDs, HTC Vive Pro with a fixed IPD of $64 \mathrm{~mm}$ and the Fove 0 (non adjustable IPD, fixed at $63 \mathrm{~mm}$ )

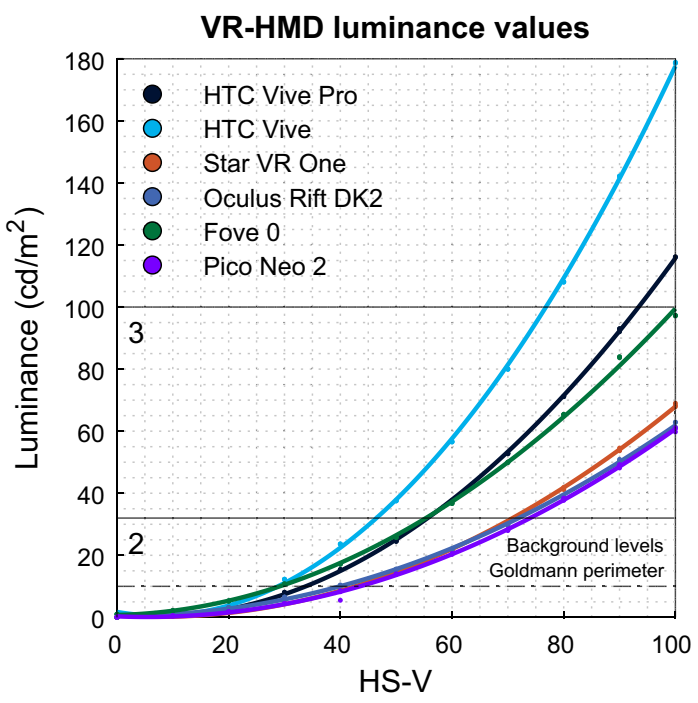

Fig. 7 Figure showcasing the luminance measured for each headset at different "Value", while at fixed Hue and Saturation

After defining the subjective FoV limits within VR, the subjects were also tested in a traditional kinetic perimeter (Octopus 900, Haag Streit International, Koeniz, Switzerland), using the same parameters (IV2e).

In the Fig. 8, the objective field of view at the average eye relief distance is plotted for both headsets, together with the average contour from the VR perimetry test and the Oculus 900 measurement.

As observed, the HTC Vive Pro does not reach the human eye limits on the nasal, temporal or lower fields of view. On the other hand, StarVR One does reach it on the upper side and overpasses the temporal side. Only nasal and inferior 
Fig. 8 Comparison between the eye's visual field limits measured using the Octopus 900 and the field of view measured in virtual reality with kinetic perimetry paradigm. Average of six subjects. Objective field of view from the StarVR and HTC Vive Pro at the subject's mean eye relief distance

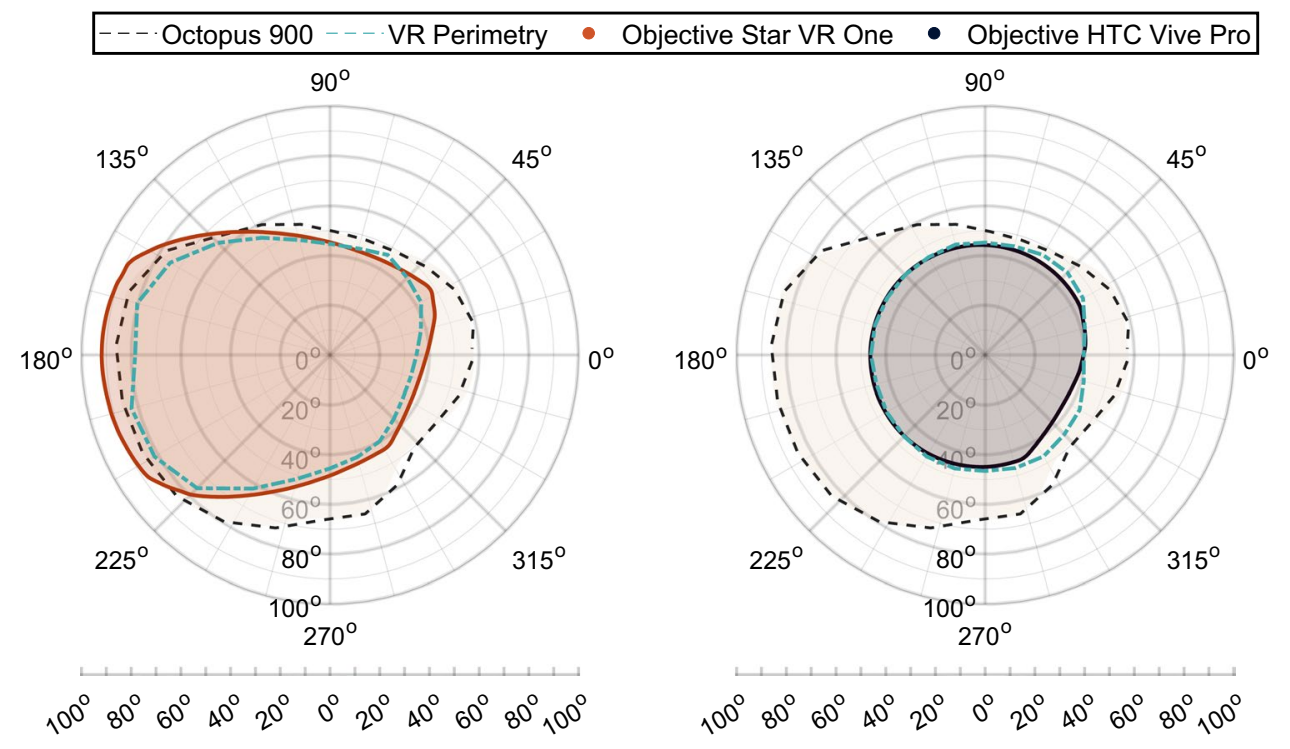

fields of view are slightly smaller than the subjects' visual field.

\section{Discussion}

This study introduces an easy and cost-effective way to measure the perceived field of view for VR headsets. Our camera-based measurement system provides a precise outline of the perceived field of view shape, reproducing eye relief and pupillary distance dependence. Based on these results, the range of expected visible fields of view can be compared with the manufacturers' specifications. Thanks to this development, it can be tested whether the use of these devices for the analysis of vision limits is valid. In contrast to other suggested methods (Lynn et al. 2020), our measurements are performed imitating common case scenarios, so all possible influencing factors (physiological, optical or screen-related) are considered.

Eye relief The distance from users' eyes to the headset lenses affects comfort while wearing these devices and modifies the area of the visual field that is exposed to the virtual content. The farther away the eyes lay from the headset lens, the smaller this area becomes. This distance was measured in multiple subjects for each headset. Results show that eye relief varies significantly among headsets, and within the same headset. This variation can result in smaller or more extensive fields of view. Therefore, tasks that require accurate stimulus positioning should measure this parameter before estimating the typical field of view covered by a certain HMD. For psychophysical experiments depending on comparable FoV sizes between subjects, we suggest using additional masking of the virtual scene to guarantee reproducible conditions for the FoV limitation .
Measuring eye relief can become complicated. Subjects may easily change how the headset rests on their faces or how tightly they fasten the straps holding it. Moreover, this parameter also varies heavily between subjects due to different facial anatomies. In this study, we propose an analog and simple method to measure this parameter. Albeit not exempt from errors, it has been proven to be accurate enough to estimate and compare the expected field of view for the average subject.

Some HMDs, such as both HTC headsets and the Rift DK2, allow physical modification of this distance, for example to enable users to wear spectacles with the HMD. The lens distance can be increased by up to $\sim 1 \mathrm{~cm}$ more than the minimum distance, which was used in this study. By doing so, eye relief increases and therefore also the size of visible FoV decreases. Consumers and researchers should be aware that adjusting the lens distance for individual subjects will lead to drastic changes in the FoV.

Interpupillary distance The adjustment of the virtual image to match the visual field of users with different IPDs has been always a topic of great relevance in virtual reality. A misalignment between the images of both eyes may cause stereopsis problems (Wann et al. 1995), inaccuracies of depth perception, convergence issues (Kramida 2016) and even diplopia if the images differ too much (Mon-Williams et al. 1993). Likewise, this difference between the central view of the lenses and the inter-pupillary distance can cause some shift on the virtual content displayed and limitations on the field of view.

Headsets including eye tracking technologies can measure the subject's individual IPD and virtually adjust the image by horizontally shifting the projection of the virtual cameras. If IPD measurement is precise, the center of the presented image should be aligned with the center of the subject's 
visual field. Some headsets, like the HTC Vive and HTC Vive Pro, additionally have an option to physically adjust the lens position to align the visual axis to the center of the lens.

Our results, simulating different IPDs, show that without adjustment of the lenses the FoV will be shifted along the horizontal axis. At some point, the screen limit is reached and the nasal or temporal FoV is cropped. Therefore, especially for users with small or big IPD, HMDs with adjustable lenses are recommended. Manual adjustment of the IPD can be a double-edged sword, as it may cause even more misalignment, if users are not aware of the adjustment option. Canted displays, like the StarVR One, do not present such big differences for IPD misalignment due to the angle toward the display.

Binocular summation The calculations for binocular field of view properties show a big overlap for all HMDs except StarVR One. For devices with circular lenses (Oculus Rift DK2 and Pico Neo 2), the FoV is almost fully symmetric, unless limited by the screen size. In this case, the overlap is almost $100 \%$. The HTC headsets and Fove 0 have a notch for the nose, reducing the size of the nasal field of view area and overlap. StarVR One has canted irregularly shaped lenses that produces an irregular binocular FoV similar to the shape of the human visual field. Given the temporal extent of the FoV this device presents, the percentage of binocular overlap is inherently smaller than other HMDs. Smaller percentage of overlap does not necessarily imply smaller overlapping area in general. Nevertheless, in this case the area seen by both eyes in the StarVR One is smaller than all other devices, due to the narrowest nasal range.

Comparison with official specifications A comparison with the manufacturers' claims is shown in Table 4. Vertical and horizontal $\mathrm{FoV}$ are always measured along meridians through the center of the visual field. In case of the StarVR One, the irregular shape of the FoV contour leads to a vertical angular distance between upper and lower contour, which is greater in some parts of the periphery than in the center. Therefore, for comparison with the product specifications, we also calculated the maximum vertical angular difference between upper and lower FoV outlines. The value for this maximum is given in Table 4 as the vertical FoV of the StarVR One.

As can be observed, every headset, measured in the course of this study, presents a smaller field of view than their official specifications suggest, even when considering the maximum binocular FoV extent along any meridian (Table 2). Only the Oculus Rift DK2 meets the manufacturer's claim at a typical eye relief distance. A brief
Table 4 Comparison of measured FoV and manufacturers' claims

\begin{tabular}{llll}
\hline HMD & $\begin{array}{l}\text { Bino. FoV (hor. } \times \\
\text { vert.) }\end{array}$ & Official specifications & Rendered (Musil 2021) \\
\hline StarVR One & $182^{\circ} \times 99^{\circ}$ & $210^{\circ} \times 130^{\circ \mathrm{a}}$ & $178^{\circ} \times 117^{\circ}$ \\
HTC Vive Pro & $94^{\circ} \times 91^{\circ}$ & $110^{\text {ob }}$ & $107^{\circ} \times 108^{\circ}$ \\
HTC Vive & $96^{\circ} \times 95^{\circ}$ & $110^{\circ \mathrm{c}}$ & $109^{\circ} \times 112^{\circ}$ \\
Pico Neo 2 & $86^{\circ} \times 88^{\circ}$ & $101^{\text {od }}$ & $101^{\circ} \times 101^{\circ}$ \\
Oculus Rift DK2 & $94^{\circ} \times 101^{\circ}$ & $100^{\circ \mathrm{e}}$ & $93^{\circ} \times 106^{\circ} *$ \\
Fove 0 & $86^{\circ} \times 92^{\circ}$ & Up to $100^{\text {of }}$ & $88^{\circ} \times 95^{\circ} *$ \\
\hline
\end{tabular}

Usually horizontal or diagonal FoV is declared. If both horizontal and vertical FoV are given by the manufacturer, it is written here as a product. The values for rendered FoV are obtained using HMDQ tool (Musil 2021), which also considers the Hidden Area Mask (HAM), a digital description of the parts of the screen which will not be seen and are not included in rendering

*For the Fove 0 and the Oculus Rift DK2, which are not included in the database, the same tool was employed to calculate this parameter

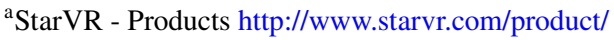

${ }^{b}$ VIVE Pro I The professional-grade VR headset

https://www.vive.com/de/product/vive-pro/

${ }^{\mathrm{c}} \mathrm{VIVE}^{\mathrm{TM}}$ I Buy VIVE Hardware

https://www.vive.com/eu/product/vive/

d Pico Neo 2

https://www.pico-interactive.com/us/neo2.html

e The All New Oculus Rift Development Kit 2 (DK2) Virtual Reality Headset

http://web.archive.org/web/20141113205611/

http://www.oculus.com/dk2/

${ }^{\mathrm{f}}$ Product FOVE Official website

https://fove-inc.com/product/

Accessed date 19/03/2021 
comparison with the rendered fields of view (Musil 2021) indicates that the manufacturers' official specifications may, in fact, refer to the rendered field of view size rather than the effective field of view. For the StarVR One, official values are even bigger than the rendered FoV. Also, during this study's measurement, the horizontal FoV extent was slightly bigger than the rendered one, which probably means that the projection of the virtual image is not precise. Such projection problems are expected since the perceived position of screen boundaries in the visual field can vary strongly with eye position. If the claimed effective FoV value is bigger than the rendered FoV, it will be accompanied by large projection errors. The visible area of the virtual world cannot stand out of the rendered area, or the projection must be distorted. Such a mismatch can be caused either by imprecise FoV settings of the virtual camera (the rendered area does not fit the HMD screen size) or by optical distortions of the lenses.

These two factors are also possible reasons for differences between the subjective test and camera-based measurements. While the subjective test uses the virtual position of a stimulus, the camera measurement finds the FoV outline in the projected image. Eye position dependence of image projection and lens distortions (Pohl et al. 2016) can lead to deviations between virtual position and perceived position. Futures studies should look at how good manufacturers apply distortion corrections to the virtual camera FoV for different eye positions within VR.

$V R$ perimetry Our subjective measurements confirm the validity of the camera-based measurement. Subjects' visual field is, as expected, larger than all traditional HMDs. Only the StarVR One's canted display panels are large enough to cover the temporal and superior part of subject's visual field. All of the tested HMDs have the potential to be used as environments for testing of central visual field loss (screening or follow-up). This confirms that these devices cover enough area to assess areas affected by typical eye diseases such as macular degeneration, glaucoma (Weber et al. 1989; Wang and Henson 2013) or retinitis pigmentosa. Only the StarVR could theoretically be used for assessing the limits of the visual field or testing for defects in the peripheral and mid-peripheral visual field areas, enabling the possibility of early detection of ocular diseases that affect the visual field.

Nonetheless, the luminance range of the tested devices limits their usage in visual field testing. The maximum brightness varied between headsets. None of the tested devices were capable of reproducing the brightest Goldmann stimulus level "4" (320 $\left.\mathrm{cd} \mathrm{m}^{-2}\right)$, which is usually used for intermediate and peripheral areas. Only the original HTC Vive was able to present the stimulus level $3\left(100 \mathrm{~cd} \mathrm{~m}^{-2}\right)$. This prevents the exact results from traditional testing methods from being reproduced in virtual perimetry.

\section{Conclusions}

In summary, manufacturers' claims regarding the field of view are not consistent with our measurements of the effectively perceived field of view. These variations appear due to the interpupillary distance and variations on the eye relief (HMD lens to eye distance). Although some manufacturers already mention that there can be variations in the field of view, it does not explicitly indicate how much is changed. These assessments regarding the field of view may ultimately lead to confusion and inaccurate assumptions for developers. We propose a new standard format for the headsets' field of view by reporting on objectively measured field of view sizes for average eye relief values, in the best case along with its typical range. Eye relief in general has to be considered as a major influence on perceived field of view. We strongly recommend that future studies that use VR technologies and require an accurate stimulus position either measure the eye relief of their subjects and estimate the field of view for that specific headset, or consider performing a subjective field of view test. They should also acknowledge eye relief differences as a potential cause of errors in their measurements.

Finally, almost every VR headset can be used for testing central visual field defects to a certain extent (similar to campimeters). Perimetry cannot be performed as peripheral areas of the field of view are usually not exposed by those headsets. Even in the case of wide-field of view devices, the screen luminance prevents testing of the intermediate and peripheral areas with traditional clinical parameters.

Supplementary Information The online version contains supplementary material available at https://doi.org/10.1007/s10055-021-00619-x.

Acknowledgements We thank the members of the workshop for building the headset holder clamps, special thanks to Herr Vollmer. We thank Melanie Kempf who made the measurements with the Octopus 900 possible and Zhijian Zhao for printing our 3D models.

Author contributions All authors contributed to the study conception and design. Material preparation, data collection and analysis were performed by Yannick Sauer and Miguel García García. The first draft of the manuscript was written by Yannick Sauer, Miguel García García and Alexandra Sipatchin. All authors commented on previous versions of the manuscript. All authors read and approved the final manuscript. Miguel García García and Siegfried Wahl, participated in the project administration and supervision. Funding acquisition was carried out by Siegfried Wahl.

Funding Open Access funding enabled and organized by Projekt DEAL. The Federal Ministry of Education and Research (BMBF) supported the project in the framework of IDeA (project number 16SV8104). Authors also recognize intra-mutual funding of the University of Tübingen through the mini graduate school 'Integrative Augmented Reality (I-AR)'. The founders did not have any additional role 
in the study design, data collection and analysis, decision to publish or preparation of the manuscript.

Availability of data and material (data transparency) The 3D models used to measure the eye relief distance can be downloaded at https:// zeissvisionsciencelab.github.io/HMD-FOV/downloads.

\section{Declarations}

Conflict of interest The authors declare that there are no conflicts of interest that could have interfered in the course of this study. Y.S., A.S. and M.G. are scientists at the University Tübingen, and S.W. is scientist at the University Tübingen and is employed by Carl Zeiss Vision International $\mathrm{GmbH}(\mathrm{E})$.

Code availability (software application or custom code) The code generated during the current study is available from the corresponding author on reasonable request.

Ethics approval The study adhered to the tenets of the Declaration of HELSINKI (2013). The ethics authorization to perform the measurements was granted by the Medicine Faculty Human Research Ethics Committee from the University of Tübingen with the ID 986/2020B02. Prior to data collection, the experiment was explained in detail to the participants, and written informed consent was collected from each participant. All data were pseudo-anonymized and stored in full compliance with the principles of the Data Protection Act GDPR 2016/679 of the European Union.

Open Access This article is licensed under a Creative Commons Attribution 4.0 International License, which permits use, sharing, adaptation, distribution and reproduction in any medium or format, as long as you give appropriate credit to the original author(s) and the source, provide a link to the Creative Commons licence, and indicate if changes were made. The images or other third party material in this article are included in the article's Creative Commons licence, unless indicated otherwise in a credit line to the material. If material is not included in the article's Creative Commons licence and your intended use is not permitted by statutory regulation or exceeds the permitted use, you will need to obtain permission directly from the copyright holder. To view a copy of this licence, visit http://creativecommons.org/licenses/by/4.0/.

\section{References}

Alexander T, Westhoven M, Conradi J (2017) Virtual environments for competency-oriented education and training, pp 23-29. https:// doi.org/10.1007/978-3-319-42070-7_3

Bashshur R, Doarn CR, Frenk JM, Kvedar JC, Woolliscroft JO (2020) Telemedicine and the COVID-19 pandemic, lessons for the future. https://doi.org/10.1089/tmj.2020.29040.rb, www.liebertpub.com

Bric JD, Lumbard DC, Frelich MJ, Gould JC (2016) Current state of virtual reality simulation in robotic surgery training: a review. Surg Endosc 30(6):2169-2178. https://doi.org/10.1007/ s00464-015-4517-y

Caramenti M, Pretto P, Lafortuna CL, Bresciani JP, Dubois A (2019) Influence of the size of the field of view on visual perception while running in a treadmill-mediated virtual environment. Front Psychol. https://doi.org/10.3389/fpsyg.2019.02344

Cipresso P, Giglioli IAC, Raya MA, Riva G (2018) The past, present, and future of virtual and augmented reality research: a network and cluster analysis of the literature. Front Psychol. https://doi. org/10.3389/fpsyg.2018.02086

Goldmann H (1946) Demonstration unseres neuen Projektionskugelperimeters samt theoretischen und klinischen Bemerkungen über Perimetrie. Ophthalmologica 111(2-3):187-192. https://doi.org/ $10.1159 / 000300322$

Hassan SE, Hicks JC, Lei H, Turano KA (2007) What is the minimum field of view required for efficient navigation? Vis Res 47(16):2115-2123. https://doi.org/10.1016/j.visres.2007.03.012

Hollander DA, Volpe NJ, Moster ML, Liu GT, Balcer LJ, Judy KD, Galetta SL (2000) Use of a portable head mounted perimetry system to assess bedside visual fields. Br J Ophthalmol 84(10):11851190. https://doi.org/10.1136/bjo.84.10.1185

Kirchner J, Watson T, Lappe M (2021) Real-time MRI reveals unique insight into the full eye kinematics of eye movements. Eneuro. https://doi.org/10.1523/ENEURO.0357-21.2021

Kramida G (2016) Resolving the vergence-accommodation conflict in head-mounted displays. IEEE Trans Visual Comput Graphics 22(7):1912-1931. https://doi.org/10.1109/TVCG.2015.2473855

Lee HS, Lew H, Yun YS (2006) Ultrasonographic measurement of upper eyelid thickness in Korean children with Epicanthus. Korean J Ophthalmol 20(2):79. https://doi.org/10.3341/kjo. 2006.20.2.79

Lynn MH, Luo G, Tomasi M, Pundlik S, Houston EK (2020) Measuring virtual reality headset resolution and field of view: implications for vision care applications. Optom Vis Sci 97(8):573582. https://doi.org/10.1097/OPX.0000000000001541

Martschinke J, Martschinke J, Stamminger M, Bauer F (2019) Gazedependent distortion correction for thick lenses in HMDs. In: 2019 IEEE conference on virtual reality and $3 d$ user interfaces (VR). IEEE, pp 1848-1851. https://doi.org/10.1109/VR.2019. 8798107

Masnadi S, Pfeil KP, Sera-Josef JVT, LaViola JJ (2021) Field of view effect on distance perception in virtual reality. In: 2021 IEEE Conference on virtual reality and 3D user interfaces abstracts and workshops (VRW). IEEE, pp 542-543. https:// doi.org/10.1109/VRW52623.2021.00153

Mees L, Upadhyaya S, Kumar P, Kotawala S, Haran S, Rajasekar S, Friedman DS, Venkatesh R (2020) Validation of a headmounted virtual reality visual field screening device. J Glaucoma 29(2):86-91. https://doi.org/10.1097/IJG.0000000000 001415

Miller HL, Bugnariu NL (2016) Level of immersion in virtual environments impacts the ability to assess and teach social skills in autism spectrum disorder. Cyberpsychol Behav Soc Netw 19(4):246-256. https://doi.org/10.1089/cyber.2014.0682

Mohamed Elias Z, Batumalai UM, Azmi ANH (2019) Virtual reality games on accommodation and convergence. Appl Ergon 81:102879. https://doi.org/10.1016/j.apergo.2019.102879

Mon-Williams M, Warm JP, Rushton S (1993) Binocular vision in a virtual world: visual deficits following the wearing of a headmounted display. Ophthalmic Physiol Opt 13(4):387-391. https:// doi.org/10.1111/j.1475-1313.1993.tb00496.x

Musil R (2021) HMD Geometry Database I Collected geometry data from some commercially available VR headsets. https://risa2000. github.io/hmdgdb/

Neugebauer A, Stingl K, Ivanov I, Wahl S (2021) Influence of systematic gaze patterns in navigation and search tasks with simulated Retinitis Pigmentosa. Brain Sci. https://doi.org/10.3390/brain sci11020223

Osuobeni EP, Al-Mijalli MH (1997) Association between eyelid thickness and corneal astigmatism. Clin Exp Optom 80(1):35-39. https://doi.org/10.1111/j.1444-0938.1997.tb04845.x

Pfandler M, Lazarovici M, Stefan P, Wucherer P, Weigl M (2017) Virtual reality-based simulators for spine surgery: a systematic 
review. The Spine Journal 17(9):1352-1363. https://doi.org/10. 1016/j.spinee.2017.05.016

Pohl D, Zhang X, Bulling A, Grau O (2016) Concept for using eye tracking in a head-mounted display to adapt rendering to the user's current visual field. In: Proceedings of the 22nd ACM conference on virtual reality software and technology. ACM, New York, NY, USA, pp 323-324. https://doi.org/10.1145/2993369.2996300,

Pretto P, Ogier M, Bülthoff HH, Bresciani JP (2009) Influence of the size of the field of view on motion perception. Computers \& Graphics 33(2):139-146. https://doi.org/10.1016/j.cag.2009.01. 003

Rolland JP, Hopkins T (1993) A method of computational correction for optical distortion in head-mounted displays. Citeseer

Sauer Y, Scherff M, Lappe M, Rifai K, Stein N, Wahl S (2021) Selfmotion illusions from distorted optic flow in multifocal glasses. iScience. https://doi.org/10.1016/j.isci.2021.103567

Scaramuzza D, Martinelli A, Siegwart R (2006) A toolbox for easily calibrating omnidirectional cameras. In: IEEE international conference on intelligent robots and systems, pp 5695-5701. https:// doi.org/10.1109/IROS.2006.282372

Segawa K, Ujike H, Okajima K, Saida S (2012) Perception of heading speed from radial flow depends on visual field. Opt Rev 19(4):268-275. https://doi.org/10.1007/s10043-012-0041-7

Sipatchin A, Wahl S, Rifai K (2021) Eye-tracking for clinical ophthalmology with virtual reality (VR): a case study of the HTC Vive Pro eye's usability. Healthcare 9(2):180. https://doi.org/10.3390/ healthcare 9020180

Stein N, Niehorster DC, Watson T, Steinicke F, Rifai K, Wahl S, Lappe M (2021) A comparison of eye tracking latencies among several commercial head-mounted displays. i-Perception 12(1):2041669520983338. https://doi.org/10.1177/2041669520 983338

Tatiyosyan SA, Rifai K, Wahl S (2020) Standalone cooperation-free OKN-based low vision contrast sensitivity estimation in VR-a pilot study. Restor Neurol Neurosci 38(2):119-129. https://doi. org/10.3233/RNN-190937

Tieri G, Morone G, Paolucci S, Iosa M (2018) Virtual reality in cognitive and motor rehabilitation: facts, fiction and fallacies. Expert Rev Med Devices 15(2):107-117. https://doi.org/10.1080/17434 440.2018.1425613

Tsapakis S, Papaconstantinou D, Diagourtas A, Droutsas K, Andreanos K, Moschos MM, Brouzas D (2017) Visual field examination method using virtual reality glasses compared with the humphrey perimeter. Clin Ophthalmol 11:1431-1443. https://doi.org/10. 2147/OPTH.S131160

Tsapakis S, Papaconstantinou D, Diagourtas A, Kandarakis S, Droutsas K, Andreanos K, Brouzas D (2018) Home-based visual field test for glaucoma screening comparison with Humphrey perimeter. Clin Ophthalmol (Auckland, NZ) 12:2597-2606. https://doi.org/ 10.2147/OPTH.S187832

Tychsen L, Foeller P (2020) Effects of immersive virtual reality headset viewing on young children: visuomotor function, postural stability, and motion sickness. Am J Ophthalmol 209:151-159. https:// doi.org/10.1016/j.ajo.2019.07.020

Urban S, Leitloff J, Hinz S (2015) Improved wide-angle, fisheye and omnidirectional camera calibration. ISPRS J Photogramm Remote Sens 108:72-79. https://doi.org/10.1016/j.isprsjprs.2015.06.005

Wang Y, Henson DB (2013) Diagnostic performance of visual field test using subsets of the 24-2 test pattern for early glaucomatous field loss. Invest Ophthalmol Vis Sci 54(1):756-761. https://doi. org/10.1167/iovs.12-10468

Wann JP, Rushton S, Mon-Williams M (1995) Natural problems for stereoscopic depth perception in virtual environments. Vision Res 35(19):2731-2736. https://doi.org/10.1016/0042-6989(95) 00018-u

Warren WH, Kurtz KJ (1992) The role of central and peripheral vision in perceiving the direction of self-motion. Perception \& Psychophysics 51(5):443-454. https://doi.org/10.3758/BF03211640

Weber J, Schultze T, Ulrich H (1989) The visual field in advanced glaucoma. Int Ophthalmol 13:47-50

Wheelwright BM, Sulai Y, Geng Y, Luanava S, Gao W, Gollier J, Gollier J (2018) Field of view: not just a number. In: Osten W, Stolle H, Kress BC (eds) Digital Optics for Immersive Displays. SPIE, p 3. https://doi.org/10.1117/12.2307303,

Zyda M (2005) From visual simulation to virtual reality to games. Computer 38(9):25-32. https://doi.org/10.1109/MC.2005.297

Publisher's Note Springer Nature remains neutral with regard to jurisdictional claims in published maps and institutional affiliations. 